\title{
PAPER
}

\section{Abnormalities of the corpus callosum in first episode, treatment naive schizophrenia}

\author{
M S Keshavan, V A Diwadkar, K Harenski, D R Rosenberg, J A Sweeney, J W Pettegrew
}

J Neurol Neurosurg Psychiatry 2002;72:757-760

See end of article for authors' affiliations

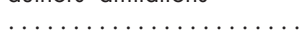

Correspondence to:

Dr M S Keshavan, Western

Psychiatric Institute and

Clinic, $3811 \mathrm{O}^{\prime}$ Hara

Street, Pittsburgh, PA

15213, USA

keshavanms@msx.upmc.edu

Received 30 May 2001

In final revised form

28 January 2002

Accepted

8 February 2002

\begin{abstract}
Background: Structural alterations in the association cortices as well as in the corpus callosum (CC) have been described in schizophrenia, and have been considered to reflect developmental abnormalities. Areas of primary and association cortices have been topographically mapped in the CC.

Objective: To investigate whether, in schizophrenia, there are alterations in CC subdivisions that connect association, but not primary, cortices, and also to see if the normative, developmentally mediated increase in CC size with age is absent in this disorder.

Methods: The midsagittal magnetic resonance imaging scans of 31 first episode, neuroleptic naive, schizophrenic patients, 12 non-schizophrenic, psychotic patients, and 31 healthy controls were compared. The total area of CC as well as that of anterior, middle and posterior genu, body, isthmus, and anterior, middle, and posterior splenii were measured.

Results: Patients with schizophrenia as a group had a smaller CC, anterior genu, anterior body, isthmus, and anterior splenium than normal controls. Furthermore, the age related increase in CC size seen in normal subjects was absent in the patients.

Conclusions: The observed reductions in size in selected regions of CC suggest a reduction in axonal connections between the heteromodal association cortices, which typically involve small diameter fibres. Furthermore, the absence of an age related increase in CC size in patients with schizophrenia suggests a neurodevelopmental abnormality that may extend into adolescence and early adulthood.
\end{abstract}

$\mathrm{T}$ he corpus callosum (CC), the major commissural pathway between the cerebral hemispheres, has been a region of much interest in schizophrenia. Early post mortem studies indicated an increased thickness of the CC in patients, ${ }^{1}$ possibly reflecting a "hyperconnection" between the hemispheres. ${ }^{2}$ In vivo imaging results have been inconclusive, although reports suggest smaller CC area schizophrenia. ${ }^{3}$ Analysis of CC subregions may be more instructive, with two lines of evidence suggesting region specific differences in CC area. Firstly, primate studies suggest a topographic mapping of cortical areas to specific CC regions ${ }^{45}$ and regional differences in the fibre composition. ${ }^{67}$ Whereas association cortical regions project via small axons $(<2 \mathrm{~mm}$ in diameter), the visual, somatosensory, primary motor, and sensory cortices project via large axons $\left(>2 \mathrm{~mm}^{8}\right)$. Secondly, schizophrenia is associated with volume reductions in heteromodal association cortices, especially the prefrontal, temporal, and inferior parietal cortex. ${ }^{9}$ Thus CC reductions are likely to be observed in the anterior genu and body (connecting prefrontal cortex), isthmus (connecting the inferior parietal cortex), and anterior splenium (connecting the superior temporal cortex) (fig 1A). ${ }^{5}$ In addition, the neurodevelopmental models of schizophrenia ${ }^{1011}$ and the fact that the CC is one of the latest maturing, highest order neural commissures of the brain, ${ }^{12}$ point to the importance of examining the relation between this structure and age in healthy and ill adolescents and young adults. The present study examined whether there are regionally and diagnostically specific CC abnormalities in first episode schizophrenia, and whether schizophrenia results in an aberration in age related alterations in CC.

\section{METHODS}

Subjects were 31 first episode, treatment naive, schizophrenia, schizoaffective, or schizophreniform disorder (SCZ) patients, 12 treatment naive patients with non-schizophrenic psychotic disorders (PSY), and 31 normal controls (NC) (table 1). They were recruited from among patients consecutively admitted to the inpatient or outpatient services of Western Psychiatric Institute and Clinic, Pittsburgh. In the SCZ group, 27 patients were diagnosed with schizophrenia, and four with schizoaffective disorder. In the PSY group, three patients were diagnosed with bipolar disorder with psychotic symptoms, four with psychosis and depression, three with psychosis (not otherwise specified), and two with delusional disorders. The patients were diagnosed by the Diagnostic and statistical manual (DSM-IV) criteria at consensus conference meetings of senior diagnostician/clinical researchers (MSK, GLH, NRS) about one month after entry into the study. The diagnosis incorporated all available clinical information and data gathered using the Structured clinical interview for DSM diagnosis (SCID), ${ }^{13}$ and were confirmed at 6-12 month follow up assessments.

Illness durations were computed (based on a consensus from SCID and all other clinical data) from the date of onset of prodromal symptoms to the date of entry into the study. Clinical ratings were carried out using the Brief psychiatric rating scale (BPRS). ${ }^{14}$ Handedness was determined by the 12 items of the Annette handedness scale, and right handedness was defined by eight more of the items being designated as right handed.

Normal control subjects were recruited by advertisement in local neighborhoods and communities in which the patient subjects reside. SCID non-patient (NP) interviews verified absence of any current or past Axis I disorder in these subjects. None of the subjects had any substance abuse or dependence (within three months), significant medical illness, or mental retardation as defined by an intelligence quotient (IQ) lower than 75. Normal controls were matched, as a group, with the schizophrenic patients for age, sex, race, and parental socioeconomic status using the Hollingshead four-factor index. ${ }^{15}$ After

Abbreviations: CC, corpus callosum; DSM, Diagnostic and statistical manual; SCID, Structured clinical interview for DSM diagnosis; MRI, magnetic resonance imaging 
A

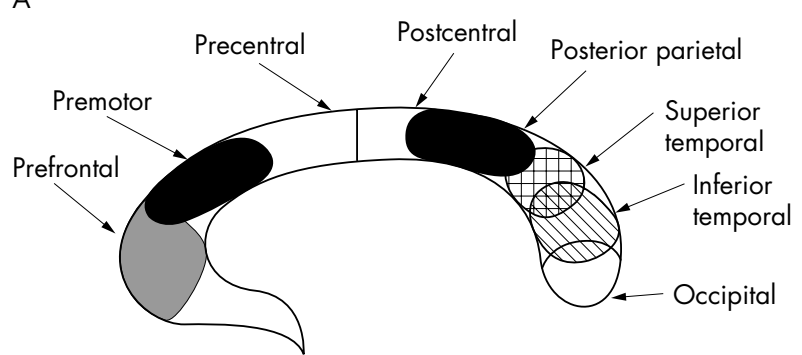

B

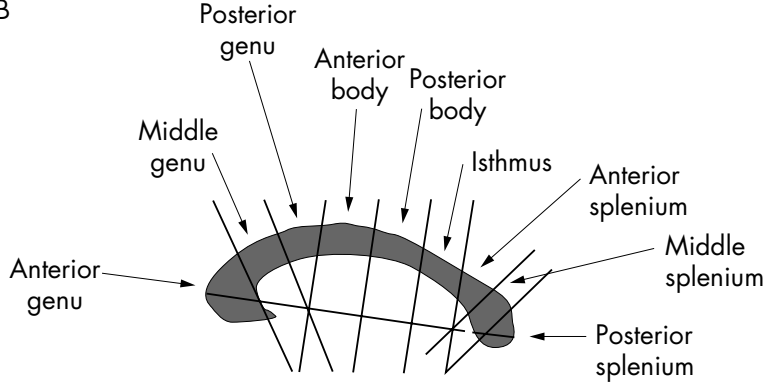

Figure 1 (A) Diagrammatic representation of commissural pathways in the corpus callosum based on neurohistological studies. ${ }^{17}$ (B) The scheme used to subdivide the corpus callosum is depicted on a sagittal view of the structure. The mapping between the division scheme used and the commissural pathways outlined in $(A)$ is clearly identifiable.

an initial telephone screening, these subjects were interviewed by a senior psychiatrist (MSK) or senior clinical psychologist (GLH) using the SCID (NP version). All subjects provided written informed consent after complete description of the study.

\section{Magnetic resonance imaging (MRI) studies}

MRI scans were conducted using a 1.5 T G.E. Signa system (G.E. Medical Systems, Milwaukee, Wisconsin, USA) at the University of Pittsburgh Medical Center, Pittsburgh, Pennsylvania, USA. Nine to $115 \mathrm{~mm}$ thick sagittal slices with a $1 \mathrm{~mm}$ interslice gap were obtained using a spin echo pulse sequence. The MRI parameters were $\mathrm{TR}=400$ milliseconds, $\mathrm{TE}=20$ milliseconds; $256 \times 256$ acquisition matrix, $\mathrm{NEX}=1, \mathrm{FOV}=20 \mathrm{~cm}$; flip angle $=10^{\circ}$. The image data were identified by scan number alone to retain blindness, and analysed using IMAGE software (version 1.55). ${ }^{16}$

\section{CC definition}

None of the MRI scans (patients or controls) showed any motion artefact. A single trained rater (WB), blind to the study hypotheses, subject identification, and clinical data, measured all scans. Identification and quantification of the CC was accomplished by selecting the midsagittal slice (the criterion was the clearest visualisation of the CC and the septum pellucidum, cerebellum and patency of the aqueduct) and was confirmed in the orthogonal coronal plane. Coronal tilt was measured in most subjects, and no differences in tilt were observed between patients and controls $(p>0.39)$, indicating that the definition of the CC did not vary across experimental groups. After this, the rater manually traced the CC along its edges. A line was drawn connecting the most anterior point of the genu with the most posterior point of the splenium (the A-P line). Using a technique adapted from Witelson, ${ }^{17}$ the CC was divided into the genu, the body, the isthmus, and the splenium. Further divisions were made to measure specific areas of the CC in a manner roughly representing CC connections hypothesised across cortical brain regions. ${ }^{45}$

Firstly a line was drawn to connect the midpoint of the A-P line within the genu with the midpoint of the line separating the genu from the body. This line was trisected by two perpendiculars, dividing the genu into its anterior, middle, and posterior regions. To divide the splenium, the midpoint of the A-P line within the splenium was connected to the midpoint of the line separating the isthmus and the splenium. This line was then trisected by two perpendiculars dividing the splenium into the anterior, middle, and posterior regions. Figure $1 \mathrm{~B}$ shows the scheme. Intracranial area was also measured on the same slice by tracing along the inner table of the skull, above the sphenoid sinus, along the basisphenoid, and across the foramen magnum. The interrater reliabilities (interclass $r$ ), based on 10 scans measured by two raters, were very high for CC measures $(r=0.97-0.99)$, intracranial area $(r=0.94)$, and ventricular volume $(r=0.99)$. Intrarater reliabilities (intraclass $r$ ), based on 10 scans measured twice by the same rater (WB) about a month apart, were also high for these structures $(r>0.96)$.

\section{Data analysis}

To protect against multiple comparisons, multiple analysis of covariance was first used to test for differences in areas in the genu, body, isthmus, and splenium, using group (SCZ, PSY, NC) and sex as independent variables, and with an $\alpha$ level set at $p<0.10$. When this criterion was met, analysis of covariance or multiple analysis of covariance (in the case of multiple subdivisions within CC regions) was used to analyse the entire CC and its constituent regions separately. Planned comparisons were then used to examine pairwise group differences (SCZ $v$ NC, SCZ $v$ PSY, PSY $v$ NC) further in CC structures. Intracranial area was used as a covariate in the analysis of all the morphometric measures. The intracranial area at the same midsagittal slice was chosen in view of a greater correlation between CC size and this measure $(r=0.41 ; \mathrm{p}<0.02)$ than $\mathrm{CC}$ size and intracranial volume $(r=0.35 ; \mathrm{p}<0.14)$. The relation between age and CC measures was assessed using multiple regression and partial correlations. Spearman correlations were used to examine the relation between illness duration and CC measures.

\begin{tabular}{|c|c|c|c|}
\hline & $\begin{array}{l}\text { Schizophrenic patients } \\
(\mathrm{n}=31)\end{array}$ & $\begin{array}{l}\text { Non-schizophrenic/ } \\
\text { psychotic patients } \\
(n=12)\end{array}$ & $\begin{array}{l}\text { Controls } \\
(\mathrm{n}=31)\end{array}$ \\
\hline Age & $24.20(8.06)$ & $22.83(5.83)$ & $25.09(6.23)$ \\
\hline $\operatorname{Sex}(M / F)$ & $20 / 11$ & $6 / 6$ & $20 / 11$ \\
\hline Handedness (R/L/mixed) & $23 / 8$ & $10 / 2$ & $28 / 3$ \\
\hline Social class & $3.42(1.41)$ & $3.17(1.53)$ & $3.87(1.50)$ \\
\hline |llness duration (weeks) & $212.09(280.91)$ & $133.25(141.90)$ & N/A \\
\hline Psychosis severity (BPRS) & $49.61(11.79)$ & $44.58(11.88)$ & N/A \\
\hline
\end{tabular}

Where applicable, values are mean (SD). There were no significant differences between groups BPRS, Brief psychiatric rating scale. 


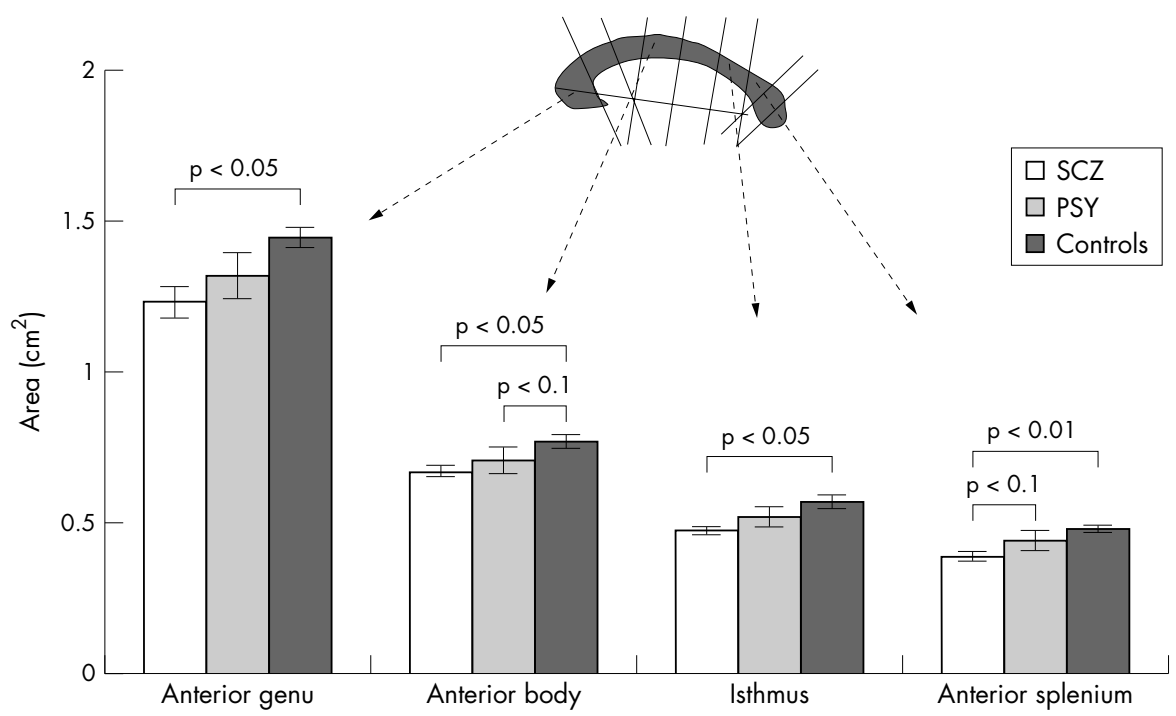

Figure 2 Size differences between experimental groups are depicted in each of four sub areas of the corpus callosum that connect the heteromodal cortices. Error bars are 1SE. Each significant or marginally significant pairwise comparison is separately indicated. SCZ, first episode, treatment naive, schizophrenia, schizoaffective, or schizophreniform disorder patients; PSY, treatment naive patients with non-schizophrenic psychotic disorders.

\section{RESULTS}

Table 1 presents basic and clinical features of the subjects. There were no significant differences between the patients and controls with regard to age, sex, or handedness. A two factor analysis of variance for intracranial area showed significant effects of sex $(F(1,68)=32.33, \mathrm{p}<0.0001)$ and group $(F(2,68)=4.67, \mathrm{p}<0.02)$, with no interaction $(\mathrm{p}>0.5)$, reflecting smaller head size in women and in schizophrenia. ${ }^{18}$

\section{CC abnormalities}

Multiple analysis of covariance showed a trend for group (Rao's $R=1.73, \mathrm{df}=(8,128), \mathrm{p}=0.097)$ and a sex by group interaction (Rao's $R=1.75, \mathrm{df}=(8,128), \mathrm{p}=0.093)$. Analysis of covariance addressing differences in total CC size showed a significant main effect of group $(F(2,67)=5.14, \mathrm{p}<0.009)$, but no effect of sex $(\mathrm{p}>0.5)$. A planned comparison showed that as a group, the SCZ group had a smaller CC area than the NC group $\left(5.75 \mathrm{~cm}^{2}\right.$ (SD 0.87) v $6.69 \mathrm{~cm}^{2}$ (SD 0.79), $F(1,67)=9.4$, $\mathrm{p}<0.003)$. Furthermore, Newman-Keuls post hoc tests showed significant differences between the other groups. The CC area in the SCZ group showed a trend for being significantly smaller than the PSY group $\left(5.75 \vee 6.19 \mathrm{~cm}^{2}\right.$ (SD $1.18), \mathrm{p}<0.07)$, and the area in the PSY group was smaller than in the normal group $\left(6.19 v 6.69 \mathrm{~cm}^{2} ; \mathrm{p}<0.05\right)$.

\section{CC subdivisions}

\section{Genu}

Significant differences between groups were confined to the anterior portion of the genu $(F(2,67)=3.55, \mathrm{p}<0.04)$. A planned contrast indicated that the size of the anterior genu in the SCZ group was smaller than in the normal controls $(1.23$ $\mathrm{cm}^{2}$ (SD 0.29) $v 1.44 \mathrm{~cm}^{2}$ (SD 0.23); $\left.F(1,67)=6.83, \mathrm{p}<0.012\right)$. Other comparisons did not reach significance.

\section{Body}

A significant difference for group was observed in the size of the anterior body $(F(2,67)=3.23, \mathrm{p}<0.05)$. A planned contrast indicated that the SCZ group had a smaller anterior body size than the NC group $\left(0.67 \mathrm{~cm}^{2}\right.$ (SD 0.09) $v 0.77 \mathrm{~cm}^{2}$ (SD 0.11 ), $F(1,67)=5.39, \mathrm{p}<0.03)$. Newman-Keuls pairwise post hoc comparisons showed a trend toward differences between the PSY and NC groups $\left(0.70 \mathrm{~cm}^{2}(\mathrm{SD} 0.16) v 0.77 \mathrm{~cm}^{2} ; \mathrm{p}<0.06\right)$.

\section{Isthmus}

The analysis of the isthmus indicated a trend for group differences $(F(2,67)=2.41, \mathrm{p}<0.10)$. A planned contrast showed a difference in isthmus size between the SCZ and NC groups $\left(0.47 \mathrm{~cm}^{2}(\mathrm{SD} 0.11) \vee 0.57 \mathrm{~cm}^{2}(\mathrm{SD} 0.14), F(1,67)=4.53\right.$, $\mathrm{p}<0.04)$.

\section{Splenium}

Significant differences between groups were confined to the anterior portion of the splenium $(F(2,65)=4.15, \mathrm{p}<0.02)$. A planned contrast indicated that as a group, SCZ had a smaller anterior splenium than NC $\left(0.39 \mathrm{~cm}^{2}\right.$ (SD 0.09$) v 0.49 \mathrm{~cm}^{2}$ (SD $0.11), F(1,65)=8.21, \mathrm{p}<0.006)$. Newman-Keuls post hoc tests showed a trend for smaller splenia in the SCZ than in the PSY group (0.39v0.44 $\mathrm{cm}^{2}$ (SD 0.11$)$; $\mathrm{p}<0.10$ ).

Figure 2 shows the significant group differences observed in each of the four subregions of the CC (anterior genu, anterior body, isthmus, and anterior splenium). As noted above, significant differences were focal to these four regions and were absent in the mid and posterior genu, the posterior body, and the mid and posterior splenium.

\section{Age related changes in the $\mathrm{CC}$}

The correlations between age and CC measures (with intracranial area partialled out) were computed separately for each group. The most striking result was that, whereas in the NC group, a significant positive correlation was seen between age and total CC size $(r=0.52 ; \mathrm{p}<0.003)$, genu $(r=0.46$; $\mathrm{p}<0.009)$, body $(r=0.41 ; \mathrm{p}<0.02)$, and splenium $(r=0.5 \mathrm{l}$; $\mathrm{p}<0.003$ ), no significant relation was seen between age and total CC size in the SCZ group $(r=-0.09, \mathrm{p}=0.63)$. The difference in the correlation coefficients for total CC area between the two groups ( $r=0.52 v r=-0.09)$ was marginally significant $(p<0.04$, one tailed). Furthermore, illness duration was not correlated with any of the CC measures in the SCZ group (Spearman $\rho<0.25 ; \mathrm{p}>0.2$ ).

\section{DISCUSSION}

Our observation of CC size reduction in first episode schizophrenia compared with both psychotic and normal controls corroborates those from a shape analysis of the CC in this group of subjects. ${ }^{19}$ Patients with schizophrenia had a smaller anterior genu, anterior body, isthmus, and anterior splenium, consistent with observed reductions (perhaps secondary to cell loss or reduced cell size) in the size of the structures that are connected by these regions of CC-that is, the prefrontal, temporal, and inferior parietal cortex in this illness. ${ }^{20}$ The absence of the expected relation between age and CC size in 
patients with schizophrenia ${ }^{12}$ is consistent with developmental models in schizophrenia. ${ }^{21}$

The modest CC reductions in non-schizophrenia patients may reflect the possible presence of some patients in this group who may ultimately receive a diagnosis of schizophrenia. Nonetheless, significant reductions (compared with normal controls) were observed in overall CC size, and the body of the structure. CC abnormalities have been reported in diverse psychiatric disorders, such as schizotypal personality disorder ${ }^{22}$ and autism, ${ }^{23}$ suggesting that CC abnormalities may be diagnostically non-specific.

As histopathological studies have failed to find differences in the density of axonal fibres in these regions of the $\mathrm{CC}^{24}{ }^{25}$ we suggest that the number of axonal fibres connecting the cortical regions across the CC may be smaller in schizophrenia. This conjecture is consistent with the "hypoconnectivity" or the "disconnection hypothesis" of the illness ${ }^{26}$ and with evidence indicating abnormal signal intensity in the CC of patients with schizophrenia. ${ }^{27}$ Recent diffusion tensor and magnetisation transfer imaging studies of the CC in schizophrenia have indicated reduced fractional anisotropy of the diffusion of water in the splenium, ${ }^{28}$ and reductions in the magnetisation transfer ratio in the genu ${ }^{29}$ in schizophrenia, indicating focally abnormal white matter in the CC. These results highlight the importance of studying the integrity of white matter in schizophrenia.

The strengths of this study include the use of a first episode, treatment naive population, the use of a psychiatric as well as a healthy control group, and the detailed subdivision of CC based on the known physiology of this structure. The findings raise interesting questions about the possible mechanisms underlying alterations in CC morphology in schizophrenia. Both genetic and non-genetic mechanisms have been postulated to underlie the pathophysiology of such abnormalities. The size and the structure of the CC may be at least partly under genetic control. ${ }^{30}{ }^{31}$ It may be that the observed reductions in CC size in patients with schizophrenia are genetically determined. Our observations of CC size reductions early in the illness, and the absence of a relation between these abnormalities and illness duration, are consistent with the possibility that callosal abnormalities have a neurodevelopmental basis. CC changes may result from a failure of development of the interhemispheric pathways, or genetically regulated excess in the elimination of interhemispheric axonal fibres. Further studies of CC morphology in familial and non-familial schizophrenic patients as well as among high risk relatives of patients with this illness are needed. Newer techniques such as magnetic resonance spectroscopy, ${ }^{32}$ diffusion tensor imaging, ${ }^{33}$ and magnetisation transfer imaging ${ }^{34}$ may also shed further light on the nature of callosal pathology in schizophrenia.

\section{ACKNOWLEDGEMENTS}

This work was supported in part by NIMH grants, MH45156, MH01372, MH46614, MH42969, MH01433, and MH45156, a Scottish Rite Schizophrenia Foundation Grant, and by funds received from the NIH/NCRR/GCRC grant no. M01 RR00056. Tanya Kisler and Werner Bagwell contributed to the morphometric measurements. We are also grateful to Gretchen L Haas and Nina R Schooler for help with the recruitment, and characterisation of the study subjects.

\section{Authors' affiliations}

M S Keshavan, V A Diwadkar, J W Pettegrew, Western Psychiatric Institute and Clinic, 3811 O'Hara Street, Pittsburgh, PA 15213, USA

K Harenski, Department of Psychiatry and Behavioral Sciences, Emory University, Atlanta, GA, USA

D R Rosenberg, Wayne State University School of Medicine, University

Health Center, 4201 St Antoine Blvd, Detroit, MI 48201, USA

J A Sweeney, Center for Cognitive Medicine, University of Illinois at Chicago, Chicago, IL, USA

\section{REFERENCES}

1 Rosenthal R, Bigelow LB. Quantitative brain measurements in chronic schizophrenia. Br J Psychiatry 1972;121:259-64.
2 David AS. Schizophrenia and the corpus callosum. Developmental, structural and functional relationships. Behav Brain Res 1994;64:203-11

3 Woodruff PWR, McManus IC, David AS. Meta-analysis of corpus callosum size in schizophrenia. J Neurol Neurosurg Psychiatry 1995;58:457-61

4 deLacoste MC, Kirkpatrick JB, Ross ED. Topography of the human corpus callosum. J Neuropathol Exp Neurol 1985;44:578-91.

5 Pandya DN, Seltzer B. The topography of commissural fibers. In: Lepore F, Ptito M, Jasper HH, eds. Two hemispheres, one brain. Functions of the corpus callosum. New York: Alan R Liss, Inc, 1986:47-73.

6 Aboitz F, Scheibel AB, Fisher RS, et al. Fiber composition of the human corpus callosum. Brain Res 1992;598: 143-53.

7 LaMantia AS, Rakic P. Cytological and quantitative characteristics of four cerebral commissures in the rhesus monkey. J Comp Neurol 1990;291:520-37.

8 Aboitz F, Scheibel AB, Fisher RS. Individual differences in brain asymmetries and fiber composition in the human corpus callosum. Brain Res 1992;598:154-61

9 Pearlson GD. Superior temporal gyrus and planum temporale in schizophrenia: A selective review. Prog Neuropsychopharmacol Biol Psychiatry 1997;21:1203-29.

10 Waddington JL. Schizophrenia: developmental neuroscience and pathobiology. Lancet 1993;341:531-6.

11 Murray RM, O'Callaghan E, Castle DJ, et al. A neurodevelopmental approach to the classification of schizophrenia. Schizophr Bull 1992:18:319-32.

12 Pujol J, Vendrell P, Junque $C$, et al. When does human brain development end? Evidence of corpus callosum growth up to adulthood. Ann Neurol 1993;34:71-5.

13 Spitzer RL, Williams JBW. Structured Clinical Interview for DSM-III-R. New York: New York State Psychiatric Institute, 1985.

14 Overall JE, Gorham DR. The Brief Psychiatric Rating Scale. Psychol Rep 1962; 10:779-82

15 Hollingshead AB. Four-factor Index of Social Status. New Haven, CT: Yale University, 1975.

16 Rasband W. NIH image manual. Bethesda, MD: National Institutes of Health, 1993

17 Witelson SF. Hand and sex differences in the isthmus and genu of the human corpus callosum. Brain 1989;1 12:799-835.

18 Andreasen NC, Arndt S, Cohen G, et al. Correction for head size. Psychiatry Res 1993;50:283

19 DeQuardo JR, Keshavan MS, Bookstein FL, et al. Landmark-based morphometric analysis of first-episode schizophrenia. Biol Psychiatry 1999;45: 1321-8.

20 Schlaepfer TE, Harris GJ, Tien AY. Decreased regional cortical gray matter volume in schizophrenia. Am J Psychiatry 1994;151:842-8.

21 Keshavan MS, Anderson S, Pettegrew JW. Is schizophrenia due to excessive synaptic pruning in prefrontal cortex? The Feinberg hypothesis revisited. J Psychiatr Res 1994;24:239-65.

22 Downhill Jr JE, Buchsbaum MS, Wei T, et al. Shape and size of the corpus callosum in schizophrenia and schizotypal personality disorder. Schizophr Res 2000;42: 193-208.

23 Egaas B, Courchesne E, Saitoh O. Reduced size of corpus callosum in autism. Arch Neurol 1995;52:794-801.

24 Casanova MF, Zito M, Bigelow LB, et al. Axonal counts of the corpus callosum of schizophrenic patients. J Neuropsychiatry Clin Neurosci 1989:1:391-93.

25 Nasrallah HA, McCalley-Whitters M, Bigelow LB, et al. A histological study of the corpus callosum in chronic schizophrenia. Psychiatry Res 1983;8:251-60.

26 Friston KJ. The disconnection hypothesis. Schizophr Res 1998; 30:115-25

27 Diwadkar VA, Dick EL, Harenski KA, et al. Abnormalities in corpus callosum signal intensity in schizophrenia. Proceedings of the International Society for Magnetic Resonance in Medicine 2001:9:430.

28 Foong J, Maier M, Clark CA, et al. Neuropathological abnormalities of the corpus callosum in schizophrenia: a diffusion tensor imaging study. $J$ Neurol Neurosurg Psychiatry 2000;68:242-4.

29 Foong J, Symms MR, Barker GJ, et al. Neuropathological abnormalities in schizophrenia: evidence from magnetization transfer imaging. Brain 2001; 124:882-92.

30 Oppenheim JS, Skerry JE, Tramo M, et al. Magnetic resonance imaging morphology of the corpus callosum in monozygotic twins. Ann Neurol 1989;26:100-4

31 Pfefferbaum A, Sullivan EV, Carmelli D. Genetic regulation of regional microstructure of the corpus callosum in late life. Neuroreport 2001;12:1677-81.

32 Keshavan MS, Stanley JA, Pettegrew JW. Magnetic resonance spectroscopy in schizophrenia: methodological issues and findings: part II. Biol Psychiatry 2000;48:369-80.

33 Lim KO, Hedehus M, Moseley M, et al. Compromised white matter tract integrity in schizophrenia inferred from diffusion tensor imaging. Arch Gen Psychiatry 1999;56:367-74.

34 Rademacher J, Engelbrecht V, Burgel U, et al. Measuring in vivo myelination of human white matter fiber tracts with magnetization transfer MR. Neurolmage 1999;9:393-406. 